\title{
A new method for investigating osteoarthritis using Fast Field-Cycling nuclear magnetic resonance
}

\author{
Lionel M. Broche ${ }^{1}$, P. James Ross ${ }^{1, *}$, Brett W.C. Kennedy, Campbell F. MacEachern, \\ David J. Lurie, George P. Ashcroft \\ Aberdeen Biomedical Imaging Centre, School of Medicine, Medical Sciences \& Nutrition, University of Aberdeen, Foresterhill, Aberdeen AB25 2ZD, UK
}

\section{A R T I C L E I N F O}

\section{Keywords:}

Fast field-cycling NMR

Human hyaline cartilage

Osteoarthritis

T1 dispersion

Quadrupolar peaks

Protein interactions

\begin{abstract}
A B S T R A C T
Osteoarthritis in synovial joints remains a major cause of long-term disability worldwide, with symptoms produced by the progressive deterioration of the articular cartilage. The earliest cartilage changes are thought to be alteration in its main protein components, namely proteoglycan and collagen. Loss of proteoglycans bound in the collagen matrix which maintain hydration and stiffness of the structure is followed by collagen degradation and loss. The development of new treatments for early osteoarthritis is limited by the lack of accurate biomarkers to assess the loss of proteoglycan. One potential biomarker is magnetic resonance imaging (MRI). We present the results of a novel MRI methodology, Fast Field-Cycling (FFC), to assess changes in critical proteins by demonstrating clear quantifiable differences in signal from normal and osteoarthritic human cartilage for in vitro measurements. We further tested proteoglycan extracted cartilage and the key components individually. Three clear signals were identified, two of which are related predominantly to the collagen component of cartilage and the third, a unique very short-lived signal, is directly related to proteoglycan content; we have not seen this in any other tissue type. In addition, we present the first volunteer human scan from our whole-body FFC scanner where articular cartilage measurements are in keeping with those we have shown in tissue samples. This new clinical imaging modality offers the prospect of non-invasive monitoring of human cartilage in vivo and hence the assessment of potential treatments for osteoarthritis. Keywords: Fast Field-Cycling NMR; human hyaline cartilage; Osteoarthritis; T1 dispersion; quadrupolar peaks; protein interactions
\end{abstract}

\section{Introduction}

Human articular hyaline cartilage is composed of a dense extracellular matrix (ECM) made up of $70 \%$ water and $30 \%$ protein. The major protein components are type II collagen and proteoglycans, with other non-collagenous proteins present in small amounts. All are regenerated slowly but continuously by chondrocyte cells through the structure [1] and it is the combination of proteoglycans held within the overall collagen structure that combines to hold water in the ECM, which is essential in the maintenance of its unique mechanical properties. The interactions between proteoglycans and collagen are elusive but known to be of electrostatic nature [2].

Though the progression of osteoarthritis is likely multifactorial, in its earliest stages it presents as a loss of proteoglycan from the extracellular matrix and progressive disruption of the collagen lattice with ultimate degradation of the underlying cartilage structure [1]. New treatments are being developed to try and slow down or reverse progression [4] but they need robust biomarkers to detect these cartilage changes early enough to assess efficacy at stages when the disease is still reversible. Magnetic resonance imaging remains one of the most promising platforms to find such biomarkers and current investigations focus on various contrast sources such as $T_{2}, T_{1 \mathrm{p}}$, dGemric, sodium, ultrashort echo time (UTE), diffusion weighted imaging (DWI), and chemical exchange saturation transfer (CEST) imaging [5], though no clear and accessible candidate has emerged yet.

Fast Field-Cycling nuclear magnetic resonance (FFC-NMR) is a wellestablished analysis technique $[2,3]$ that can measure the variations of the spin-lattice relaxation time constant, $T_{1}$ with the magnetic field strength, also called the $T_{1}$ dispersion curve, or $R_{1}$ dispersion curve where $R_{1}=1 / T_{1}$. In FFC-NMR the typical measurement process is comprised of three stages - polarisation, evolution and detection with magnetic field strength $\left(B_{0}\right)$ values $B_{0}^{\mathrm{P}}, B_{0}^{\mathrm{E}}$ and $B_{0}^{\mathrm{D}}$ respectively (see

\footnotetext{
* Corresponding author.

E-mail address: james.ross@abdn.ac.uk (P. James Ross).

1 Dr. Broche and Dr. Ross are joint first authors.
} 


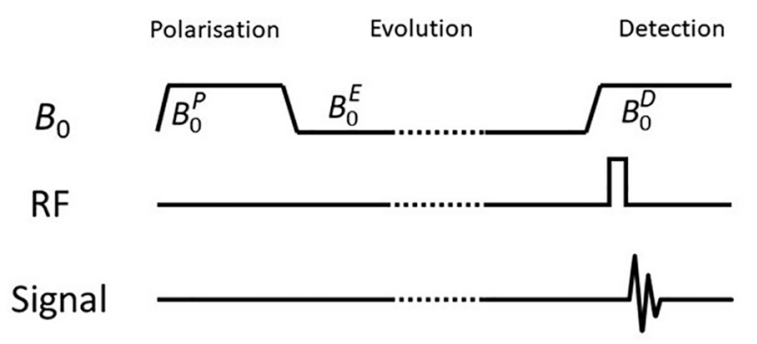

Fig. 1. Example of a basic Fast Field-Cycling Pulse sequence showing the three main periods: Polarisation, Evolution and Detection. During Polarisation the sample magnetisation is built up in a relatively strong magnetic field $\left(B_{0}^{\mathrm{P}}\right)$. During Evolution the magnetic field is switched to a value of interest $\left(B_{0}^{\mathrm{E}}\right)$ and the sample undergoes spin-lattice relaxation at that field. Finally, during the Detection period the NMR signal is read out at field $B_{0}^{\mathrm{D}}$.

Fig. 1). During polarisation the sample is polarised at a fixed magnetic field - typically the highest the device can achieve - in order to generate a large sample magnetisation $\left(M_{\mathrm{Z}}\right)$. During evolution the magnetic field is rapidly switched to a new magnetic field and the magnetisation relaxes towards a new equilibrium value (as $M_{0} \propto B_{0}$ ) with associated time constant $T_{1}^{\mathrm{E}}$. Finally, during detection the magnetic field is slewed to the value corresponding to the tuned frequency of the radiofrequency coil and the NMR signal is recorded. Crucially, only the field strength during the evolution period is varied during an experiment while the signal is always detected at the same field strength. In this way $T_{1}$ information can be probed over a wide range of different magnetic fields using a single radiofrequency coil.

It is well established that $T_{1}$ varies with the magnetic field strength and that these variations are directly related to the molecular dynamics in the sample observed [4]. In biological samples, the signal observed comes mainly from water molecules so that FFC-NMR provides quantitative information about the dynamics of these water molecules. In addition to the general $T_{1}$ dispersion information, many biological tissues also exhibit so-called Quadrupolar Peaks (QP, see Fig. 2) that are due to interactions between water protons and amine or amide groups from reticulated proteins, enabling potential quantitation of proteins of interest [3].

A previous pilot study from our group suggested significant variations in both QP and dispersion curve between healthy and osteoarthritic cartilage [5]. Further investigations by other groups [6,7] have since provided more information about the QP in cartilage but the exact

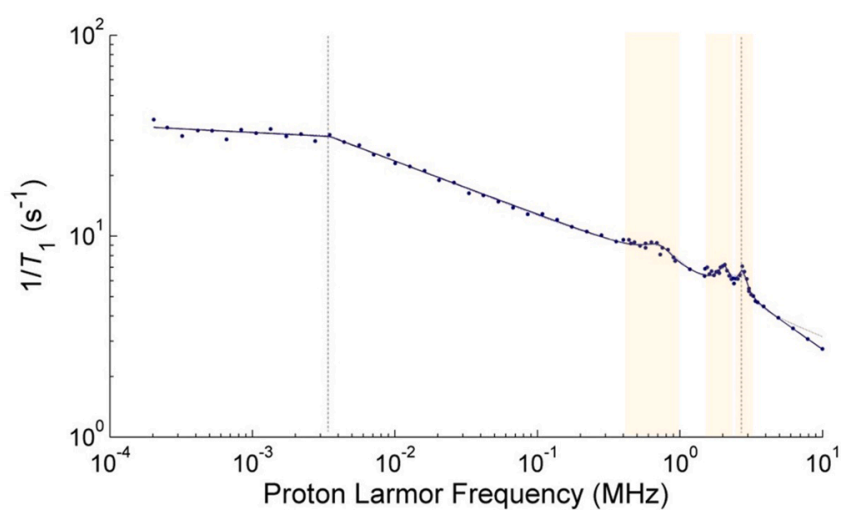

Fig. 2. Example of an $R_{1}$ dispersion curve from healthy femoral head cartilage. The general downward trend follows piecewise segments of lines, which in a logarithm plot correspond to power laws, with sudden changes of regimes shown by vertical dashed lines (here around $3 \mathrm{kHz}$ and $2.8 \mathrm{MHz}$ ). Three relaxation regimes appeared, the high-field segment is difficult to see by eye but is required for a good fit, as can be seen by extending the second segment (fine dashed line). Quadrupolar Peaks are visible around 0.7, 2.1 and 2.8 MHz PLF (yellow regions). origin remains unknown. This study aimed to investigate the in vitro behaviour of the $T_{1}$ dispersion curve and its quadrupolar signal in normal and osteoarthritic human cartilage samples using a benchtop device to identify potential biomarkers, and to understand their origins, for use in future in vivo imaging. This is particularly relevant, as our research group has successfully built a whole-body Fast Field-Cycling Imaging (FFC-imaging) scanner [8] which is now available to scan volunteers and patients. We therefore also present initial in vivo scan results from the articular cartilage of a volunteer knee. (Note that magnetic fields may be reported in equivalent Proton Larmor Frequency (PLF), where $1 \mathrm{~T}=42.5 \mathrm{MHz}$ PLF.)

\section{Materials and methods}

\section{Normal and osteoarthritic human cartilage samples}

For this study we harvested $8 \mathrm{~mm}$ diameter cartilage cores of 1-2 mm thickness from human femoral heads, femoral condyles and tibial plateau removed at surgery. Non-pathological cartilage was obtained from femoral neck fracture patients or vasculopathic patients following transfemoral amputation where there was no history or radiological evidence of arthritis. Osteoarthritic cartilage came from patients undergoing hip or knee replacement for primary osteoarthritis. The tissue samples were vacuum packed for transportation and for ease of manipulation and tissue preservation. A layer of subchondral bone of up to $1 \mathrm{~mm}$ was left attached to the core to ensure that the entire thickness of cartilage was being analysed; previous experiments confirmed that this had negligible influence on the results because NMR signal from the subchondral bone is negligible compared to that of cartilage. The cores were placed in NMR tubes with fluorinert (FC-70, Sigma) to avoid evaporation during Fast Field-Cycling NMR analyses. Samples that could not be analysed directly were stored at $4{ }^{\circ} \mathrm{C}$ for up to 5 days. This study was approved by the local North of Scotland Ethics Committee (NoSREC, www.nres.npsa.nhs.uk).

\section{Proteoglycan-depleted cartilage and purified compounds}

Additional cores of cartilage were analysed to study the effect of proteoglycan depletion. These samples were taken from five osteoarthritis patients at three different sites: immediately next to a significant area of osteoarthritis, and from more normal load bearing and non-load bearing regions on the opposite side of the joint. The dispersion curves of each core was acquired by FFC-NMR then the samples were washed briefly with phosphate-buffered saline (PBS) and placed in an extraction buffer to remove proteoglycans (4 $\mathrm{M} \mathrm{GuCl}$ with protease inhibitor) for $72 \mathrm{~h}$ at $4{ }^{\circ} \mathrm{C}$ with occasional shaking [9]. The extraction buffer was then removed, the samples washed briefly with PBS and reanalysed by FFCNMR relaxometry. The extraction of proteoglycan was confirmed by sodium dodecyl sulfate polyacrylamide gel electrophoresis (SDS-PAGE) using $100 \mu \mathrm{l}$ of the extraction buffer.

We also measured the $T_{1}$ dispersion of the extracted buffer, crosslinked hyaluronic acid ( $20 \mathrm{mg} / \mathrm{mL}$, Durolane, Q-Med AB, Sweden) and a lyophilised collagen matrix derived from bovine achilles tendon (Collatamp EG, Syntacoll GmBH Germany). Collagen matrices were prepared by humidifying dried samples with $50 \% \mathrm{w} / \mathrm{w}$ distilled water in order to increase the NMR signal without solubilising the compounds, which would have destroyed the quadrupolar peak signal.

\section{Fast Field-Cycling nuclear magnetic resonance}

All FFC-NMR measurements were performed at $37 \pm 0.1{ }^{\circ} \mathrm{C}$ using a commercial relaxometer (Stelar, Italy, SMARtracer) that can produce a maximum field strength of approximately $0.23 \mathrm{~T}$ (10 MHz PLF) with a slew rate of $200 \mathrm{~T} / \mathrm{s}$. $T_{1}$ was measured at 30 values of field strength spaced logarithmically from $230 \mathrm{mT}$ to $230 \mu \mathrm{T}$ (10 MHz to $10 \mathrm{kHz}$ PLF) with 35 points for finer sampling of the quadrupolar peaks ( 30 points 
from 1.5 to $3.5 \mathrm{MHz}$ and 5 from 0.4 to $0.9 \mathrm{MHz}$ ).

Early in the investigation we identified differences related to acquisition times and identified short- and long-lived signals. The duration of the short-lived signal was measured using a spin echo sequence and showed a $T_{2}$ of the order of $500 \mu$ s. Additional $T_{2}$ measurements at $170 \mathrm{mT}(7.4 \mathrm{MHz}$, the acquisition field) using CPMG echo trains showed a clear bi-exponential behaviour that could be fitted using $T_{2}$ values of $12.5 \pm 0.7 \mathrm{~ms}$ and $2.26 \mathrm{~ms} \pm 0.7 \mathrm{~ms}\left(\mathrm{R}^{2}\right.$-value $>0.99$ ), suggesting multiple water compartments. Therefore we used two pulse sequences to measure the $T_{1}$ dispersion in cartilage: a long acquisition sequence consisting of an inversion recovery preparation followed by a Carr-Purcell-Meiboom-Gill (CPMG) echo train (1000 echoes, $20 \mu$ s echo time, $20 \mathrm{~ms}$ total acquisition time) and a fast acquisition sequence consisting of an inversion recovery preparation followed by a saturation pulse and a $500 \mu$ s acquisition.

The detection of the NMR signal was always performed at the same magnetic field (174 mT or equivalently 7.4 MHz PLF) so that $T_{2}$ was constant throughout the experiment, regardless of the evolution field that was being investigated. $T_{2}$ measurements could therefore be performed from CPMG experiments but this proved difficult with $T_{2}$ values below $1 \mathrm{~ms}$ because of sequence limitations. Short $T_{2}$ measurements were therefore performed using a spin echo sequence with variable echo time and a $500 \mu$ s saturation acquisition.

It should be noted that our group have not observed large signals with $T_{2}$ values in the range of $500 \mu \mathrm{s}$ in any other biological tissue we have examined to date, although our studies focus mostly on soft tissues.

\section{FFC-NMR data analysis}

The data were processed using MATLAB 2014b (The Mathworks, Natick, U.S.A) using a script developed in-house to import the raw data and generate the dispersion curve. The $T_{1}$ dispersion curves were fitted using a piecewise power-law model derived from polymer studies $[2,3,6]$ in which each segment captures a different type of molecular motion. Previous results from our first pilot study indicated that the dispersion curve was adequately fitted using a three-segment power law to model the background. This provided the slope of each segment $\left(\alpha_{\text {high }}\right.$, $\alpha_{\text {mid }}$ and $\left.\alpha_{\text {low }}\right)$, the transition frequencies between these segments $\left(\nu_{\text {high }}\right.$ and $\left.\nu_{\text {low }}\right)$ and the overall vertical offset of the dispersion $\left(R_{1}\right.$ at $\left.1 \mathrm{MHz}\right)$. Note that large slopes in the dispersion curve are usually associated with significant restriction of mobility.

In addition, a model for the quadrupolar peaks published by Fries and Belorizky [10] was added, with minor modifications to avoid error propagation (normalisation of the amplitude). The quadrupolar peaks model provided the amplitude of the peaks $(A)$, the frequency of the quadrupolar transition $\left(\nu_{\mathrm{Q}}\right)$, the angular direction of the electric field gradient at the ${ }^{14} \mathrm{~N}$ nuclei responsible for the quadrupolar peaks $(\theta$ and $\varphi$ ), its asymmetry parameter $(\eta)$ and the timescale of the proton exchange at the quadrupolar site $\left(\tau_{1}\right)$. The amplitude of the quadrupolar peaks is directly proportional to the concentration of relaxation sites $[11,12]$.

Statistical analyses were performed using either $t$-tests when the noise was Gaussian-distributed or Wilcoxon signed-rank tests when a distribution could not be established. The amplitude of the quadrupolar peaks was estimated using the integral of the data from 1.8 to $3.2 \mathrm{MHz}$ after subtraction of the background by curve fitting. The amplitude of the QP was estimated from the integral of the peak amplitude, which was found to be proportional to the amplitude of the high-frequency peak with a scaling factor of 0.73 but provided lower noise.

\section{Results}

All the dispersion curves from cartilage samples showed clear linear trends when displayed in logarithmic plots (see Fig. 2) with transitions around $50 \mathrm{mT}$ ( $2 \mathrm{MHz}$ PLF) and $100 \mu \mathrm{T}(4.2 \mathrm{kHz}$ PLF). They all exhibited quadrupolar peaks around 0.7, 2.2 and 2.9 MHz PLF with very little variation in their shape other than their amplitude. Using the average from the entire dataset of 203 samples, the model provided the following parameters: $\eta=0.429 \pm 0.003, \nu_{\mathrm{Q}}=805 \pm 4 \mathrm{kHz}, \theta=82^{\circ} \pm 1^{\circ}, \varphi-32^{\circ} \pm$ $2^{\circ}$ and $\tau_{1}=0.92 \pm 0.02 \mu$ s. These parameters correspond well with the $\mathrm{N}$ (2) nuclei of $N$-glycyl L- $\alpha$ Alanine [13] and is likely to come from amino acids located at the termination of protein backbones as previously suggested in other publications $[2,3,10,14]$. Data below $230 \mu \mathrm{T}(10 \mathrm{kHz})$ were discarded because measurements below Earth's field required precise compensation methods which were not implemented at the time of this work.

\section{Results from short and long acquisitions}

Despite the apparent similarity in shape amongst all the dispersion curves, significant differences were observed depending on the acquisition method (Table 1 and Fig. 3). Long acquisitions using CPMG showed significant variation in the dispersion slope between healthy and diseased hip cartilage, both above and below $50 \mathrm{mT}\left(\Delta \alpha_{\text {high }}=\right.$ $-0.0428 \pm 0.001, \mathrm{p}<10^{-4}$, and $\Delta \alpha_{\text {mid }}=-0.0207 \pm 0.001, \mathrm{p}=0.003$ respectively, all comparison tests here were derived from t-tests) but a similar trend was not observed in the knee samples. Short acquisitions using saturation pulses showed a significant change in the slope of the dispersion in hip cartilage $\left(\Delta \alpha_{\text {high }}=+0.0646 \pm 0.001, \mathrm{p}<10^{-3}\right.$ and $\left.\Delta \alpha_{\text {mid }}=+0.0271 \pm 0.001, \mathrm{p}=0.003\right)$.

The transition frequency between the two segments, $\nu_{\text {high }}$, also changed with osteoarthritis in the hip in longer acquisitions $\left(\Delta \nu_{\text {high }}=\right.$ $-43 \mathrm{kHz}, \mathrm{p}<10^{-6}$ ), again with no significant differences in knee samples. No significant change was observed in the transition frequency in the short acquisition.

Finally, longer acquisitions showed very large differences in the vertical offset of the dispersion curve both in the hip $\left(-3.09 \pm 0.03 \mathrm{~s}^{-1}\right.$, $\left.\mathrm{p}<10^{-6}\right)$ and knee samples $\left(-0.80 \pm 0.01 \mathrm{~s}^{-1}, \mathrm{p}<10^{-3}\right)$, as did the short acquisition $\left(-3.15 \pm 0.038 \mathrm{~s}^{-1}, \mathrm{p}<10^{-6}\right)$.

Compared with short acquisitions, longer acquisitions showed less pronounced quadrupolar peaks ( $-0.16 \mathrm{~s}^{-1}, \mathrm{p}=0.012$, Fig. 4) and were less sensitive to osteoarthritis in both knee and hip samples. Quadrupolar peaks measured by short acquisitions however showed very significant differences in the quadrupolar peak amplitudes with osteoarthritis $\left(-1 \mathrm{~s}^{-1}, \mathrm{p}<10^{-5}\right)$.

\section{Proteoglycan-depleted cartilage and short $T_{2}$ signal}

In order to test the effect of Proteoglycan loss on the shortest $T_{2}$ signal, FFC-NMR measurements were performed on samples of cartilage before and after proteoglycan extraction. These samples were taken immediately adjacent to osteoarthritic lesions, and from more normal appearing cartilage at a weightbearing site and a non-weightbearing site at the opposite side of the joint. Validation by protein assay showed that the proteoglycan extraction process was more effective in osteoarthritis samples, probably because of collagen disorganisation aiding extraction [15]. The blots of the extract showed clear bands corresponding to large proteins (Fig. 5), validating the protein extraction process. Cartilage cores that were proteoglycan-depleted showed a decrease in their quadrupolar peak amplitude as measured using a fast acquisition (Fig. 6), reaching about $-80 \% \pm 20 \%$ in regions close to osteoarthritis lesions $(\mathrm{p}<0.001)$ and $-40 \% \pm 15 \%$ in the contralateral regions $(\mathrm{p}<$ 0.01). Quadrupolar peak amplitude after extraction reached quadrupolar peak amplitudes of the order of $1 \mathrm{~s}^{-1}$, which is close to what is observed from knee osteoarthritis cartilage when using long signal acquisitions (see Table 1).

\section{Characterisation of collagen, extracted proteoglycans and hyaluronic acid}

As part of the study we examined the major components of articular cartilage separately. As expected, the dispersion curve of collagen solution showed no quadrupolar peaks [14] but organised collagen, in the 
Table 1

Parameters obtained from the long and short acquisitions (median values, error bars are the standard deviation).

\begin{tabular}{|c|c|c|c|c|c|c|}
\hline \multirow[t]{2}{*}{ Parameter } & \multicolumn{2}{|c|}{ Hip cartilage, long signal } & \multicolumn{2}{|c|}{ Hip cartilage, short signal } & \multicolumn{2}{|c|}{ Knee cartilage, long signal } \\
\hline & Normal & Osteoarthritis & Normal & Osteoarthritis & Normal & Osteoarthritis \\
\hline Number of samples & 30 & 39 & 23 & 26 & 30 & 55 \\
\hline Integrated Quadrupolar Peaks amplitude $\left[\mathrm{s}^{-1} . \mathrm{MHz}\right]$ & $1.0 \pm 0.6$ & $1.0 \pm 0.4$ & $2.7 \pm 0.5$ & $1.7 \pm 0.4$ & $1.2 \pm 0.5$ & $1.1 \pm 0.3$ \\
\hline$\alpha_{\text {high }}$ & $-0.33 \pm 0.03$ & $-0.37 \pm 0.03$ & $-0.47 \pm 0.05$ & $-0.39 \pm 0.05$ & $-0.34 \pm 0.05$ & $-0.34 \pm 0.07$ \\
\hline$\alpha_{\text {mid }}$ & $-0.18 \pm 0.04$ & $-0.20 \pm 0.04$ & $-0.30 \pm 0.03$ & $-0.27 \pm 0.04$ & $-0.22 \pm 0.03$ & $-0.20 \pm 0.04$ \\
\hline$\nu_{\text {high }}[\mathrm{MHz}]$ & $2.7 \pm 0.6$ & $2.2 \pm 0.4$ & $1.8 \pm 0.3$ & $1.8 \pm 0.1$ & $2.3 \pm 0.6$ & $2.0 \pm 0.5$ \\
\hline Vertical offset $\left[\mathrm{s}^{-1}\right]$ & $11 \pm 2$ & $8.4 \pm 1.1$ & $12.1 \pm 1.2$ & $9.4 \pm 0.9$ & $8.8 \pm 1.2$ & $8.1 \pm 0.9$ \\
\hline
\end{tabular}



Fig. 3. Typical NMRD profiles of the same sample of healthy human cartilage using the long and short acquisition protocols. Differences can be seen in the slopes and amplitude of the quadrupolar peaks, which are quantified in Table 1.

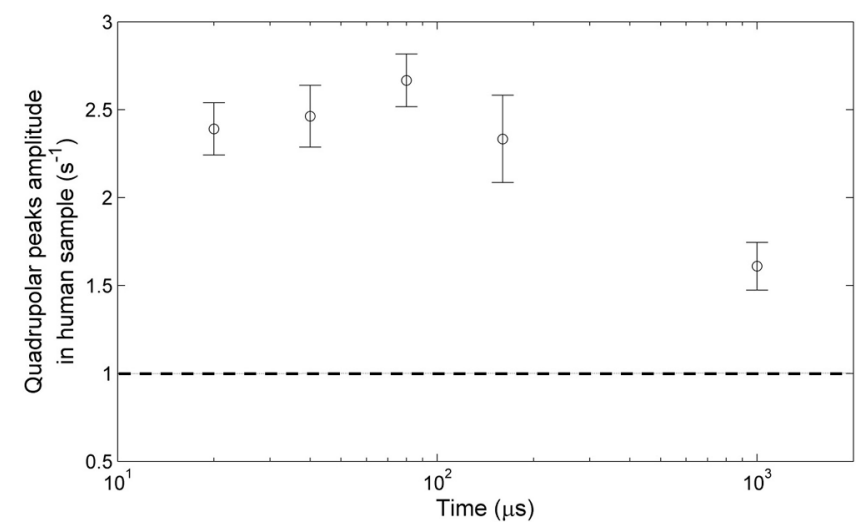

Fig. 4. Evolution of the Quadrupolar Peaks amplitude with the delay between the excitation pulse and the data acquisition. The amplitude obtained from the long acquisition is shown by the horizontal dashed line. The duration of the signal of interest for the characterisation of osteoarthritis seems to be on the order of hundreds of microseconds.

collagen matrix used in this study, exhibited visible quadrupolar signal, although much lower than seen in articular cartilage (see Fig. 7). The dispersion curves obtained from $50 \% \mathrm{w} / \mathrm{w}$ collagen matrices exhibited only the longest $T_{2}$ signals and showed integrated quadrupolar peak amplitudes of $0.17 \pm 0.04 \mathrm{~s}^{-1}$, corresponding to a peak height of $0.23 \pm$ $0.05 \mathrm{~s}^{-1}$, or $0.46 \mathrm{~s}^{-1}$ for $1 \mathrm{~g} / \mathrm{mL}$ of collagen, in agreement with published work [7]. The $T_{1}$ dispersion curves also showed a 2-component behaviour $\left(\nu_{\text {high }}=-0.25 \pm 0.02\right.$ and $\left.\nu_{\text {mid }}=-0.157 \pm 0.004\right)$ with a transition at $1.8 \pm 0.3 \mathrm{MHz}$. The hyaluronic acid gel and proteoglycan extracted from cartilage cores did not show quadrupolar peaks, even though other work has suggested that concentrated hyaluronic acid solutions may

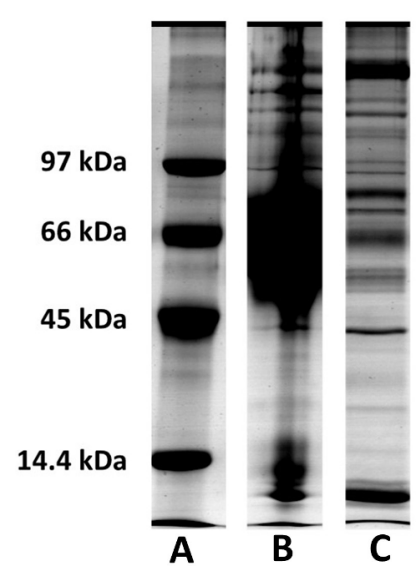

Fig. 5. SDS PAGE gels of the extraction buffers obtained from the contralateral (b) and osteoarthritis lesion cartilage samples (c). The molecular markers are shown on the left for reference (a).

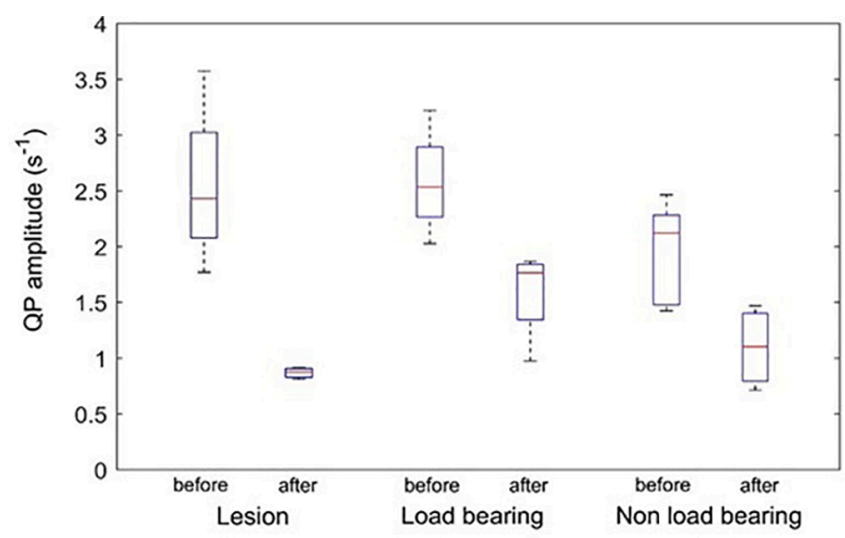

Fig. 6. Average amplitude of the high-frequency Quadrupolar Peaks for the different cores before and after proteoglycan extraction using a GuCl buffer. A marked decrease is clearly visible with the extraction process and the Quadrupolar Peaks amplitude reached after extraction is similar to that observed from long acquisitions in the knee (note the factor 0.73 between Quadrupolar Peaks amplitude and integrated Quadrupolar Peaks amplitude).

lead to weak quadrupolar peaks in the dispersion curve [7].

\section{Discussion}

This study suggests that the signals obtained from the $T_{1}$ dispersion curve using FFC have real potential for the quantitative measurement of the proteoglycan loss and collagen matrix degradation seen in osteoarthritic articular cartilage. The largest osteoarthritis-related changes observed in the $T_{1}$ dispersion curve were related to the amplitude of the quadrupolar peak, the vertical shift of the dispersion curve and the slope of the dispersions in the log-log plot. 


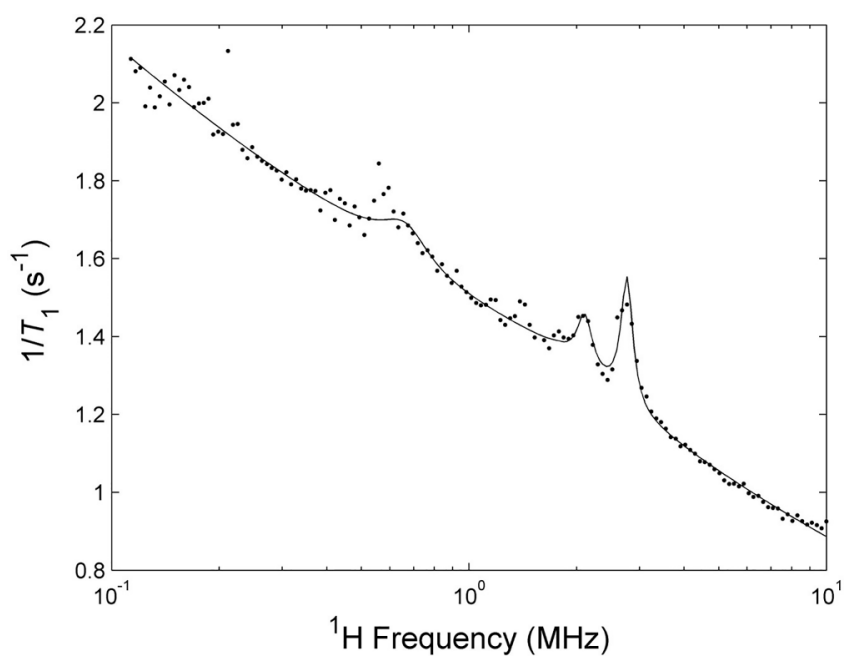

Fig. 7. Dispersion curve of humidified collagen matrix at $50 \% \mathrm{w} / \mathrm{w}, 37{ }^{\circ} \mathrm{C}$. Large Quadrupolar Peaks are visible (Integrated Quadrupolar Peaks amplitude of $0.17 \pm 0.04 \mathrm{~s}^{-1}$, offset $=1.48 \pm 0.01 \mathrm{~s}^{-1}, \eta=0.41 \pm 0.01, \nu_{\mathrm{Q}}=0.812 \pm$ $\left.0.005 \mathrm{MHz}, \theta=79 \pm 9^{\circ}, \varphi=26 \pm 6^{\circ}, \tau_{1}=1.1 \pm 0.2 \mu \mathrm{s}\right)$ and the background showed a 2-component behaviour $\left(\alpha_{\text {high }}=-0.25 \pm 0.02\right.$ and $\alpha_{\text {low }}=-0.157 \pm$ 0.004 , transition at $1.8 \pm 0.3 \mathrm{MHz}$ ).

The identification of three different $T_{2}$ components in cartilage at the low fields employed in FFC NMR is in keeping with experiments performed at high field, where protons from pools of free water are thought to account for longer $T_{2}$ values and water bound to macromolecules for shorter $T_{2}$ components. However, the $500 \mu$ s short- $T_{2}$ signal we observed in this study has not been observed by our group in any other proteinrich biological tissues such as muscle or liver, and its amplitude is large compared to the relatively low protein concentration in cartilage (about $30 \%$ protein to $70 \%$ water). The $T_{1}$ dispersion profiles associated with the various $T_{2}$ components as shown in Table 1 indicated different types of molecular dynamics and varied in OA hip cartilage depending on the length of the acquisition. Long acquisitions, associated with medium and long $T_{2}$ values, showed an increase in the $T_{1}$ dispersion with osteoarthritis while short acquisitions showed a decrease of the dispersion with osteoarthritis. $T_{1}$ dispersion is strongly related to water mobility and the larger the dispersion, the more constrained the proton pool is likely to be. This would indicate that the proton pool associated with the shortest $T_{2}$ component is more mobile as osteoarthritis progresses while the pools from the longer $T_{2}$ components are more tightly constrained, at least in the hip. One can also see that $T_{1}$ dispersion is higher in the shortest- $T_{2}$ component, so that its associated proton pool is much less mobile than for the other two pools in general.

The increase in $T_{1}$ dispersion with osteoarthritis in hip cartilage may be due to the increased water fraction that penetrates collagen fibre bundles as their structure degrades [16] but such a mechanism would also increase water access to ${ }^{14} \mathrm{~N}$ relaxation sites and increase protein mobility, both having large but opposing effects on quadrupolar peaks amplitude. However, no changes were detected in the amplitude of quadrupolar peaks from long- $T_{2}$ signals in both hip and knee cartilage. It seems unlikely that these two non-linear effects would compensate so precisely, so we hypothesise that this increased dispersion is due to collagen fibres collapsing into a slightly denser network with shorter collagen strands accumulating inside the cartilage matrix. Such degraded collagen fibres could be too mobile to contribute to the quadrupolar peaks amplitude, similar to gelatine collagen [17], but could increase $T_{1}$ dispersion by a confinement effect. Additionally, the small but significant decrease of the transition frequency with osteoarthritis, together with the large decrease in the dispersion offset, also suggest the presence of more mobile elements in diseased cartilage and may be a biomarker of cartilage degradation though this effect must disappear at higher magnetic field when bulk water relaxation becomes predominant. In any case, collagen dynamics are likely to be a later indicator of osteoarthritis, as would be expected since collagen degradation happens later in the disease process.

Interpretations of the short-lived signals data: Quadrupolar relaxation and $T_{1}$ dispersion

The shortest $T_{2}$ component seems to be related to collagenproteoglycan interactions, which shows interesting prospects as an early biomarker of osteoarthritis. The results obtained from purified collagen and hyaluronic acid provide some insights about quadrupolar peak relaxation mechanisms when compared to that of cartilage. Structured collagen in the collagen matrix exhibited clear quadrupolar peaks but their amplitude could not account for what was observed in human cartilage. Indeed, data from long acquisitions showed that quadrupolar peaks relaxivity for lyophylised collagen was $0.46 \mathrm{~s}^{-1} / \mathrm{g}$, which was lower than for pure collagen [7], whereas it reached about 6 $\mathrm{s}^{-1} / \mathrm{g}$ in osteoarthritis cartilage if one considers that collagen is the main source of quadrupolar peaks relaxation in human cartilage. This result is supported by similar figures reported by other workers $[7,18]$. The large difference in quadrupolar peaks amplitude between articular cartilage material and purified collagen cannot be explained by the contribution from isolated hyaluronic acid, which is the second most abundant compound in human cartilage after collagen, because its quadrupolar peaks relaxivity is even weaker than that of collagen, as supported by [7], and its concentration in cartilage is even lower. Similarly, other proteoglycan components in cartilage are unlikely to bring significant contributions to quadrupolar peaks, at least when taken individually, because they account only for a small fraction of the cartilage matrix and quadrupolar peaks amplitude scales linearly with protein concentration.

According to the model developed by Fries and Belorizky [10], the large change in quadrupolar peaks amplitude can reasonably be attributed to three potential sources in cartilage: a strong decrease in the mobility of collagen when proteoglycan are present; a better access of water molecules to the ${ }^{14} \mathrm{~N}$ relaxation site due to collagen-proteoglycan interactions; or the appearance of many additional ${ }^{14} \mathrm{~N}$ relaxation sites either in proteoglycan or collagen due to their interactions. Additionally, the results obtained from collagen matrices or hyaluronic acid gel did not show short- $T_{2}$ components, indicating that the contribution in quadrupolar peaks signal from short-lived signals in healthy cartilage is not a pool of collagen or hyaluronic acid protons, or that these protons do not provide quadrupolar peaks relaxation without collagenproteoglycan interaction.

The analysis of the $T_{1}$ dispersion also shows potential information on collagen-proteoglycan interactions. The proton pool associated with the shortest $T_{2}$ showed large $T_{1}$ dispersion values, hence very low mobility, but the dispersion decreased with osteoarthritis so these protons are less confined as the disease progresses and they cannot originate from the presence of collagen alone, since the associated signal was not observed in purified collagen matrices. This pool cannot be protons from bulk water either since these would have much larger $T_{2}$ values, and it disappears with osteoarthritis and proteoglycan extraction. Yet proteoglycan extracts did not show a short-lived component, neither did hyaluronic acid gels, so it cannot be linked to proteoglycan in isolation. Hence, we hypothesise that this very short $T_{2}$ proton pool originates from water molecules trapped by hyperosmotic proteoglycan chains and stabilised by collagen-proteoglycan interactions, involving electrostatic interactions between aggrecan chains and collagen alanine amino acids that offer additional ${ }^{14} \mathrm{~N}$ relaxation to that proton pool and generates the large quadrupolar peaks observed in human cartilage from short acquisitions.

This explanation agrees with the observations made in the dispersion and quadrupolar peaks values of cartilage and with other works from the literature [19]. If this is the case, it should be visible in cartilage at silent stages of the osteoarthritis when collagen structures are relatively intact 
but proteoglycan chains are degraded or diffusing away from the cartilage matrix. We hypothesise that it may be possible to detect this in in-vivo human studies using low-field MRI or FFC-imaging systems, such as those developed by our team $[8,20]$.

\section{Conclusions}

We have demonstrated that $T_{1}$ dispersion is a promising candidate as a new biomarker in the assessment of osteoarthritis in articular cartilage. This is of particular interest for clinical research using our FFC imaging system and opens new avenues for early detection trials using this new technology [8]. We observe significant differences in features of $T_{1}$ dispersion between healthy and arthritic cartilage in excised samples, and we have shown for the first time that it is possible to access this information in vivo. The features derived from the short-lived signal $T_{1}$ dispersion component in cartilage are particularly intriguing and we have offered suggestions on a physiological origin behind these signals. If these hypotheses are correct, the detection of quadrupolar peaks would be an indirect measurement of the collagen-proteoglycans interactions, which could detect osteoarthritis at silent and potentially reversible stages. Extracting information on this component in vivo will likely be technically challenging owing to the short $T_{2}(<1 \mathrm{~ms})$ and future work will concentrate on translating recent advances in UTE techniques into FFC-imaging in order to make this possible.

\section{Declaration of Competing Interest}

The authors declare that they have no known competing financial interests or personal relationships that could have appeared to influence the work reported in this paper.

\section{Acknowledgements}

We acknowledge ARUK (Versus Arthritis) for funding this study (grant number 19869) and the NHS Grampian Biorepository for the access to cartilage samples. BWCK received a Foulkes Foundation Fellowship. This project has also received funding from the European Union's Horizon 2020 research and innovation programme under grant agreement No. 668119 (project "IDentIFY") and NHS Grampian endowments. This work received support from the EURELAX COST Action CA15209, supported by COST (European Cooperation in Science and Technology).

\section{Appendix A. Supplementary data}

Supplementary data to this article can be found online at https://doi.

\section{org/10.1016/j.ejmp.2021.05.034.}

\section{References}

[1] Pearle AD, Warren RF, Rodeo SA. Basic science of articular cartilage and osteoarthritis. Clin Sports Med 2005. https://doi.org/10.1016/j.csm.2004.08.007.

[2] Kruk D, Herrmann A, Rössler EA. Field-cycling NMR relaxometry of viscous liquids and polymers. Prog Nucl Magn Reson Spectrosc 2012;63:33-64. https://doi.org/ 10.1016/j.pnmrs.2011.08.001.

[3] Kimmich R, Anoardo E. Field-cycling NMR relaxometry. Prog Nucl Magn Reson Spectrosc 2004. https://doi.org/10.1016/j.pnmrs.2004.03.002.

[4] Kruk D. In: Understanding spin dynamics. Pan Stanford Publishing Pte. Ltd.; 2015. https://doi.org/10.1201/b19179.

[5] Broche LM, Ashcroft GP, Lurie DJ. Detection of osteoarthritis in knee and hip joints by fast field-cycling NMR. Magn Reson Med 2012;68:358-62. https://doi.org/ $10.1002 / \mathrm{mrm} .23266$.

[6] Rössler EA, Stapf S, Fatkullin N. Recent NMR investigations on molecular dynamics of polymer melts in bulk and in confinement. Curr Opin Colloid Interface Sci 2013; 18:173-82. https://doi.org/10.1016/j.cocis.2013.03.005.

[7] Rössler E, Mattea C, Stapf S. NMR dispersion investigations of enzymatically degraded bovine articular cartilage. Magn Reson Med 2015;73:2005-14. https:// doi.org/10.1002/mrm.25292.

[8] Broche LM, Ross PJ, Davies GR, MacLeod MJ, Lurie DJ. A whole-body Fast FieldCycling scanner for clinical molecular imaging studies. Sci Rep 2019;9:1-11. https://doi.org/10.1038/s41598-019-46648-0.

[9] Chascall V, Calabro A, Midura RJ, Yanagishita M. Isolation and characterization of proteoglycans. Methods Enzymol 1994. https://doi.org/10.1016/0076-6879(94) 30026-7.

[10] Fries PH, Belorizky E. Simple expressions of the nuclear relaxation rate enhancement due to quadrupole nuclei in slowly tumbling molecules. J Chem Phys 2015. https://doi.org/10.1063/1.4926827.

[11] Jiao X, Bryant RG. Noninvasive measurement of protein concentration. Magn Reson Med 1996;35:159-61.

[12] Broche LM, Ismail SR, Booth NA, Lurie DJ. Measurement of fibrin concentration by fast field-cycling NMR. Magn Reson Med 2012;67:1453-7. https://doi.org/ 10.1002/mrm.23117.

[13] Edmonds DT, Speight PA. Nitrogen quadrupole resonance in amino acids. Phys Lett A 1971;34:325-6. https://doi.org/10.1016/0375-9601(71)90894-2.

[14] Sunde EP, Halle B. Mechanism of $1 \mathrm{H}-14 \mathrm{~N}$ cross-relaxation in immobilized proteins. J Magn Reson 2010;203:257-73. https://doi.org/10.1016/j. jmr.2010.01.008.

[15] Brandt KD. Enhanced extractability of articular cartilage proteoglycans in osteoarthrosis. Biochem J 1974;143:475-8. https://doi.org/10.1042/bj1430475.

[16] Kadler KE, Holmes DF, Trotter JA, Chapman JA. Collagen fibril formation. Biochem J 1996;316:1-11. https://doi.org/10.1042/bj3160001.

[17] Kozlov PV, Burdygina GI. The structure and properties of solid gelatin and the principles of their modification. Polymer 1983. https://doi.org/10.1016/00323861(83)90001-0.

[18] Ravera E, Fragai M, Parigi G, Luchinat C. Differences in dynamics between crosslinked and non-crosslinked hyaluronates measured by using fast field-cycling relaxometry. ChemPhysChem 2015. https://doi.org/10.1002/cphc.201500446.

[19] Bittersohl B, Miese FR, Hosalkar HS, Herten M, Antoch G, Krauspe R, et al. T2* mapping of hip joint cartilage in various histological grades of degeneration. Osteoarthritis Cartilage 2012. https://doi.org/10.1016/j.joca.2012.03.011.

[20] Lurie DJ, Aime S, Baroni S, Booth NA, Broche LM, Choi C-H, et al. Fast field-cycling magnetic resonance imaging. Comptes Rendus Phys 2010. https://doi.org/ 10.1016/j.crhy.2010.06.012. 